\title{
About SSD
}

\author{
Dongjun Shin
}

Samsung Electronics 


\section{Outline}

- SSD primer

- Optimal I/O for SSD

- Benchmarking Linux FS on SSD

- Case study: ext4, btrfs, xfs

- Design consideration for SSD

- What's next?

- New interfaces for SSD

- Parallel processing of small I/O 


\section{SSD Primer (1/2)}

- Physical unit of flash memory

- Page $_{\text {NAND }}$ - unit for read \& write

- Block $_{\text {NAND }}$ - unit for erase (a.k.a erasable block)

- Physical characteristics

- Erase before re-write

- Sequential write within an erasable block

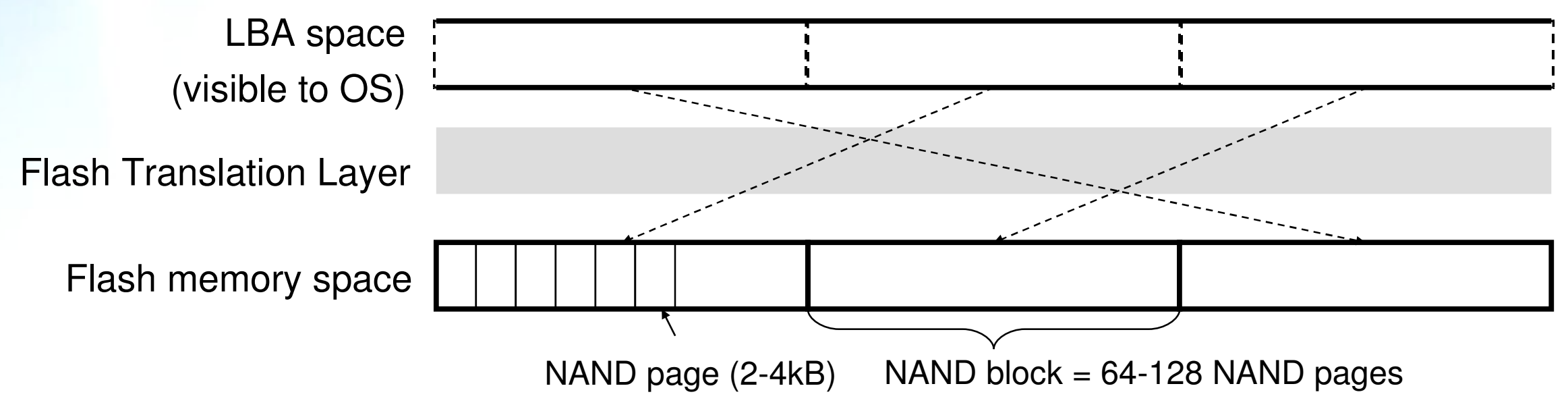




\section{SSD Primer (2/2)}

- Internal organization: 2-dimensional (NxM parallelism)

- Similar to RAID-0 (stripe size = sector or page NAND $_{\text {) }}$

- Effective page \& block size is multiplied by NxM (max)

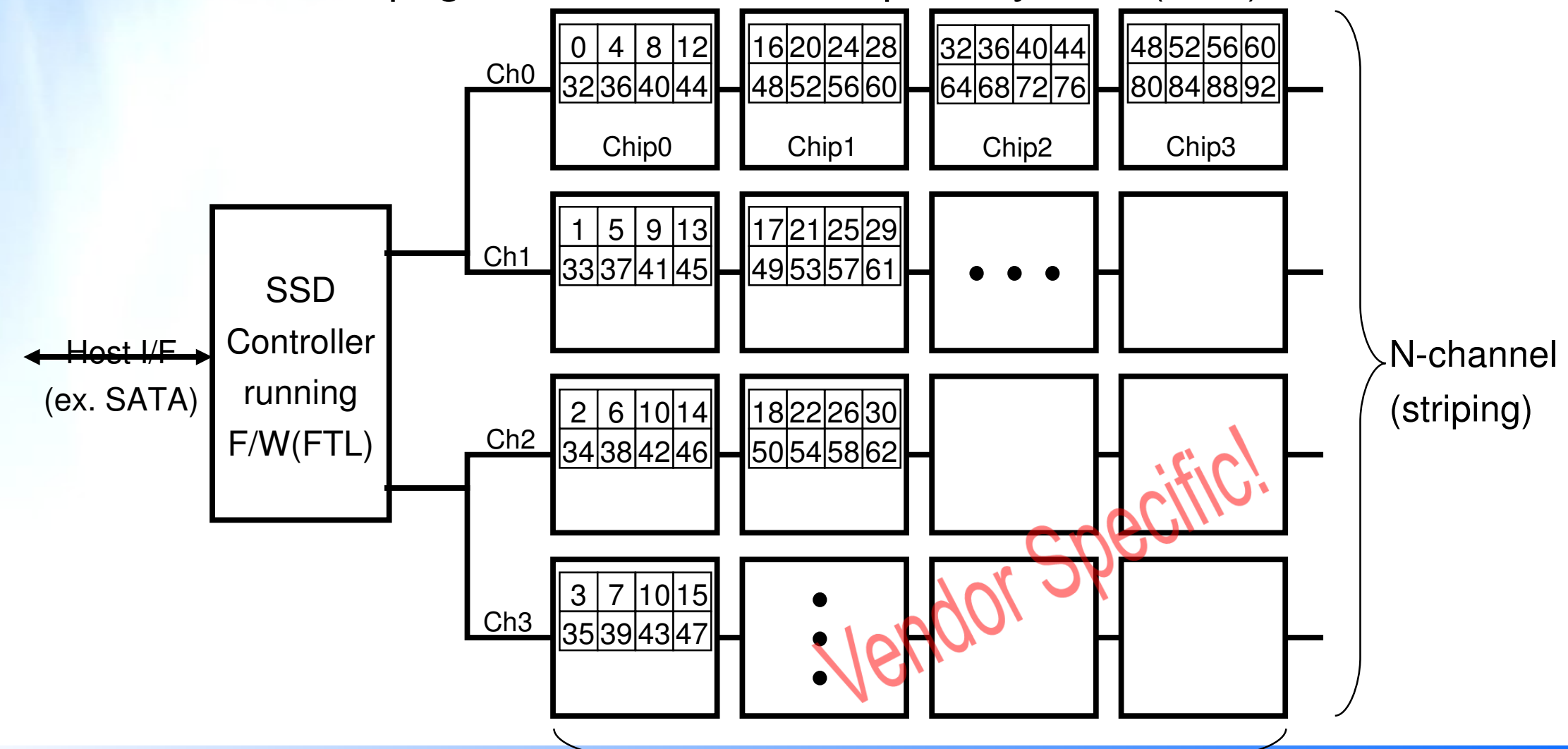




\section{Optimal I/O for SSD}

- Key points

- Parallelism

- The larger the size of I/O request, the better

- Match with physical characteristics

- Alignment with page or block size of NAND*

- Segmented sequential write (within an erasable block)

- What about Linux?

- HDD also favors larger I/O $\rightarrow$ read-ahead, deferred aggregated write

- Segmented FS layout $\rightarrow$ good if aligned with erasable block boundary

- Write optimization $\rightarrow$ FS dependent (ex. allocation policy)

* Usually, partition layout is not aligned (1st partition at LBA 63) 


\section{Test environment (1/2)}

- Hardware

- Intel Core 2 Duo E6550@2.33GHz, 1GB RAM

- Software

- Fedora 7 (Kernel 2.6.24)

- Benchmark: postmark

- Filesystems

- No journaling - ext2

- Journaling - ext3, ext4, reiserfs, xfs

- ext3, ext4: data=writeback,barrier=1[,extents]

- xfs: logbsize=128k

- COW, log-structured - btrfs (latest unstable, 4k block), nilfs (testing-8)

- SSD

- Vendor M (32GB, SATA): read $100 \mathrm{MB} / \mathrm{s}$, write $80 \mathrm{MB} / \mathrm{s}$

- Test partition starts at LBA 16384 (8MB, aligned) 


\section{Test environment (2/2)}

- Postmark workload

- Ref: Evaluating Block-level Optimization through the IO Path (USENIX 2007)

\begin{tabular}{|l|l|l|l|l|}
\hline Workload & File size & $\begin{array}{l}\text { \# of file } \\
\text { (work-set) }\end{array}$ & $\begin{array}{l}\text { \# of } \\
\text { transaction }\end{array}$ & $\begin{array}{l}\text { Total app } \\
\text { read/write }\end{array}$ \\
\hline SS & $9-15 \mathrm{~K}$ & 10,000 & 100,000 & $630 \mathrm{M} / 755 \mathrm{M}^{*}$ \\
\hline SL & $9-15 \mathrm{~K}$ & 100,000 & 100,000 & $600 \mathrm{M} / 1.8 \mathrm{G}$ \\
\hline LS & $0.1-3 \mathrm{M}$ & 1,000 & 10,000 & $9.7 \mathrm{G} / 12 \mathrm{G}$ \\
\hline LL & $0.1-3 \mathrm{M}$ & 4,250 & 10,000 & $9 \mathrm{G} / 17 \mathrm{G}$ \\
\hline \multicolumn{5}{|r}{} \\
* Mostly write-only
\end{tabular}




\section{Benchmark results (1/2)}

- Small file size (SS, SL)

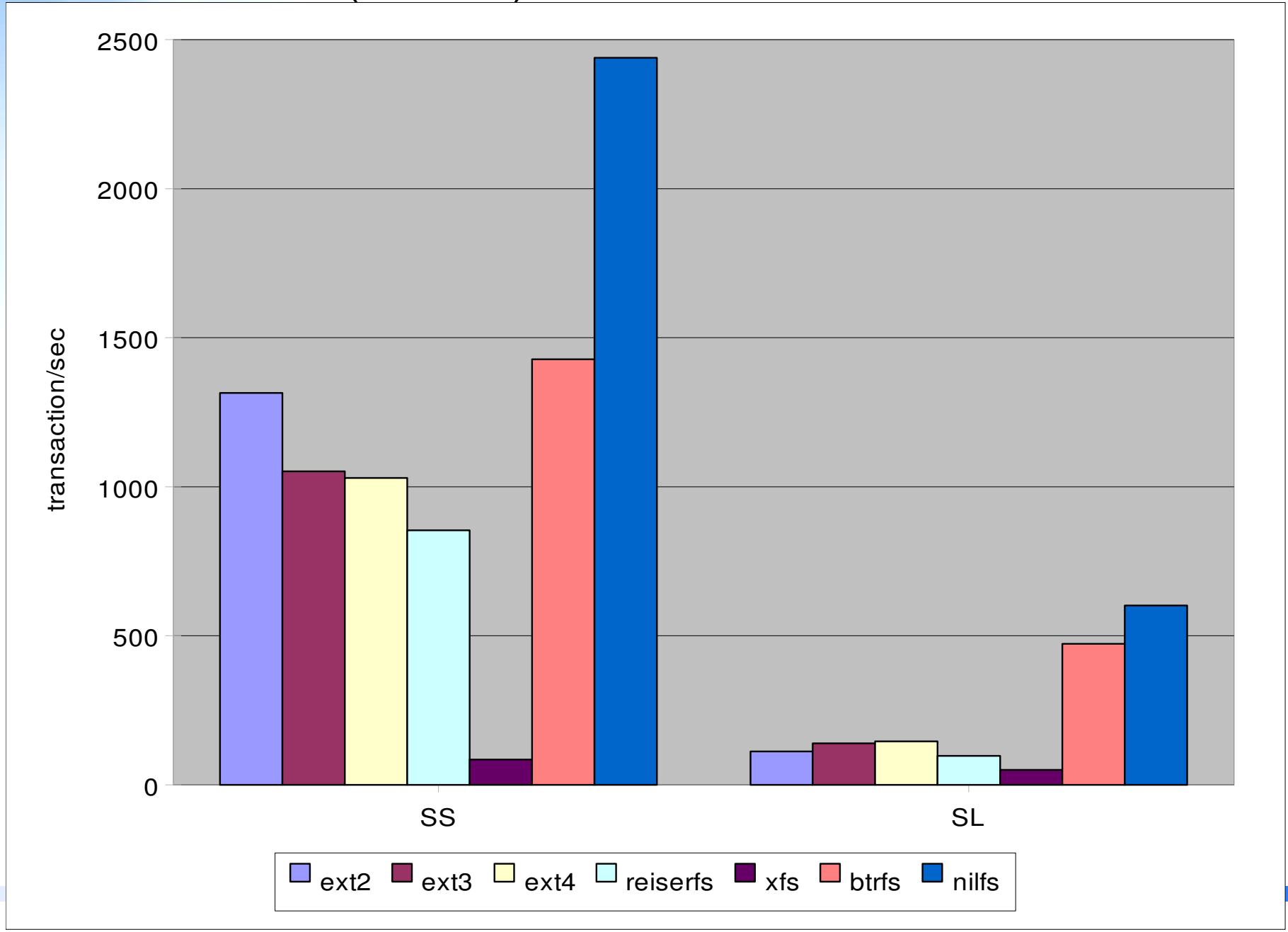




\section{Benchmark results (2/2)}

- Large file size (LS, LL)

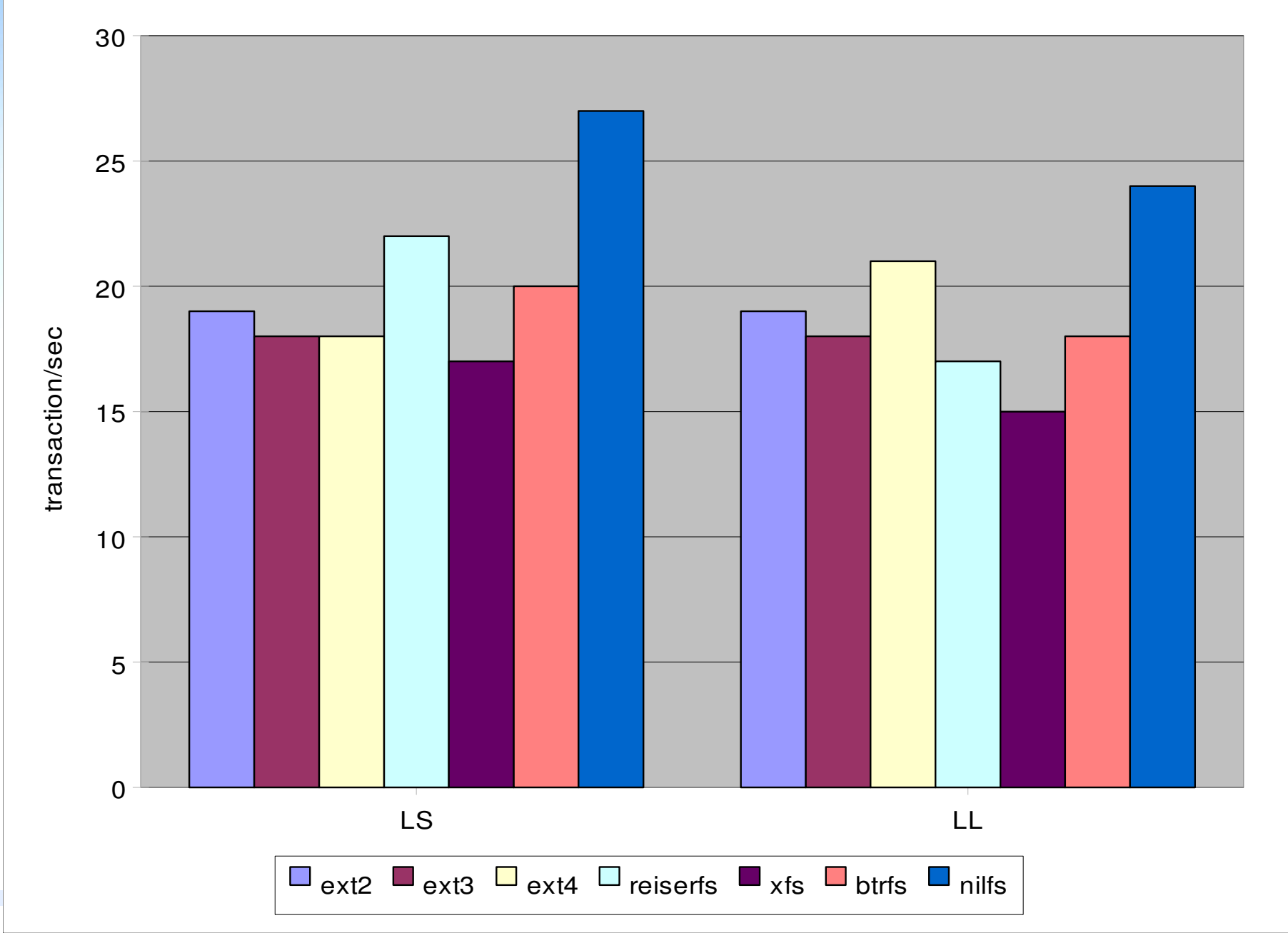




\section{I/O statistics (1/2)}

- Average size of I/O

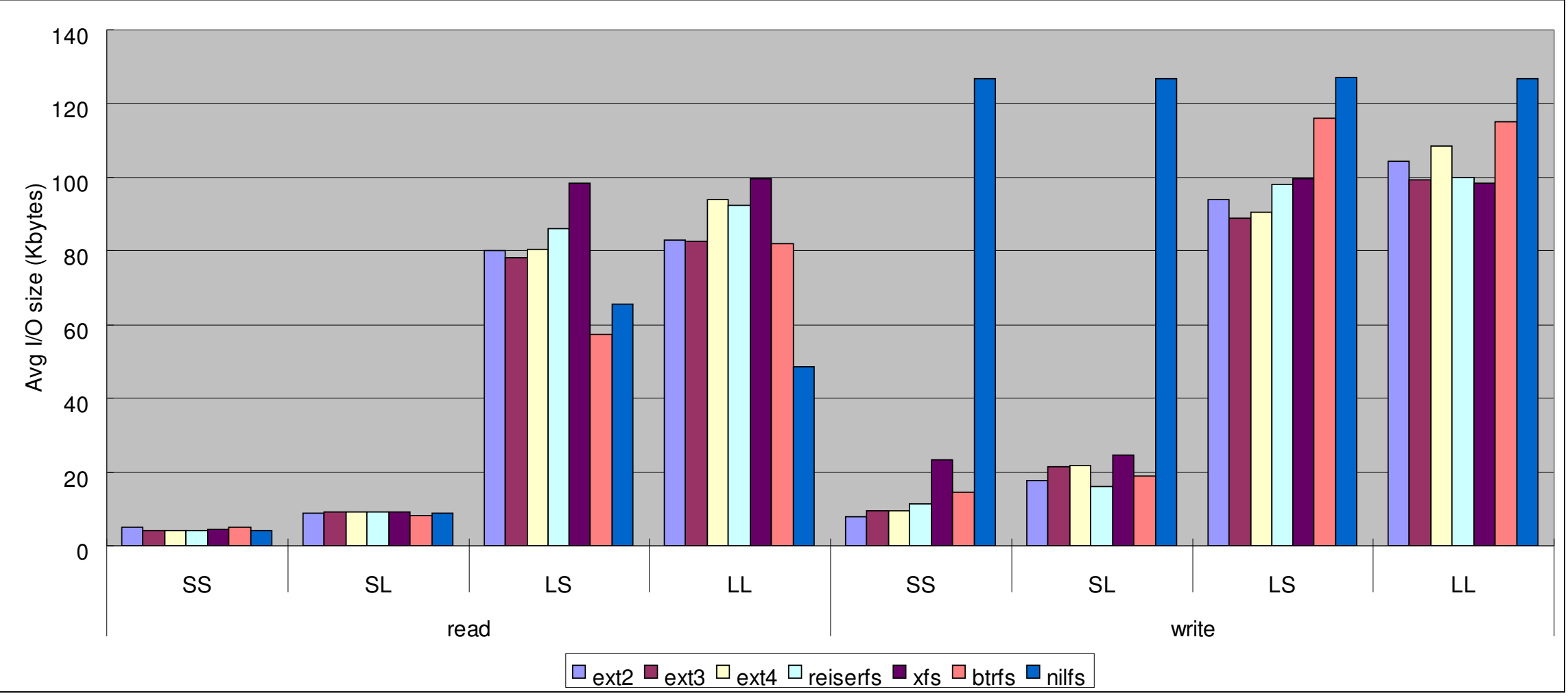




\section{I/O statistics (2/2)}

- Segmented sequentiality of write $\mathrm{I} / \mathrm{O}$ (segment: $1 \mathrm{MB}$ )

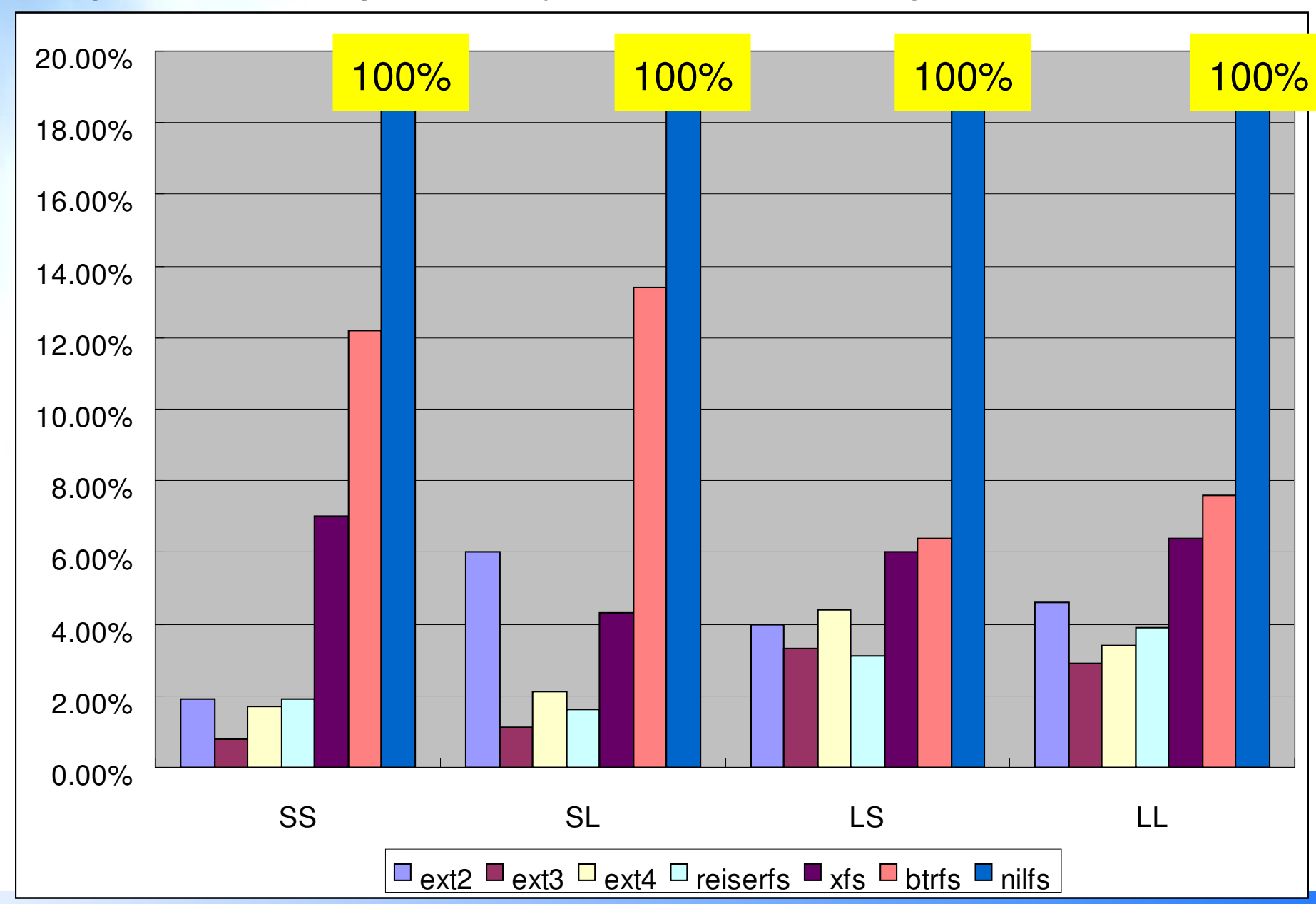




\section{Case study - ext4}

- Condition

- data=ordered, allocation: default/noreservation/oldalloc

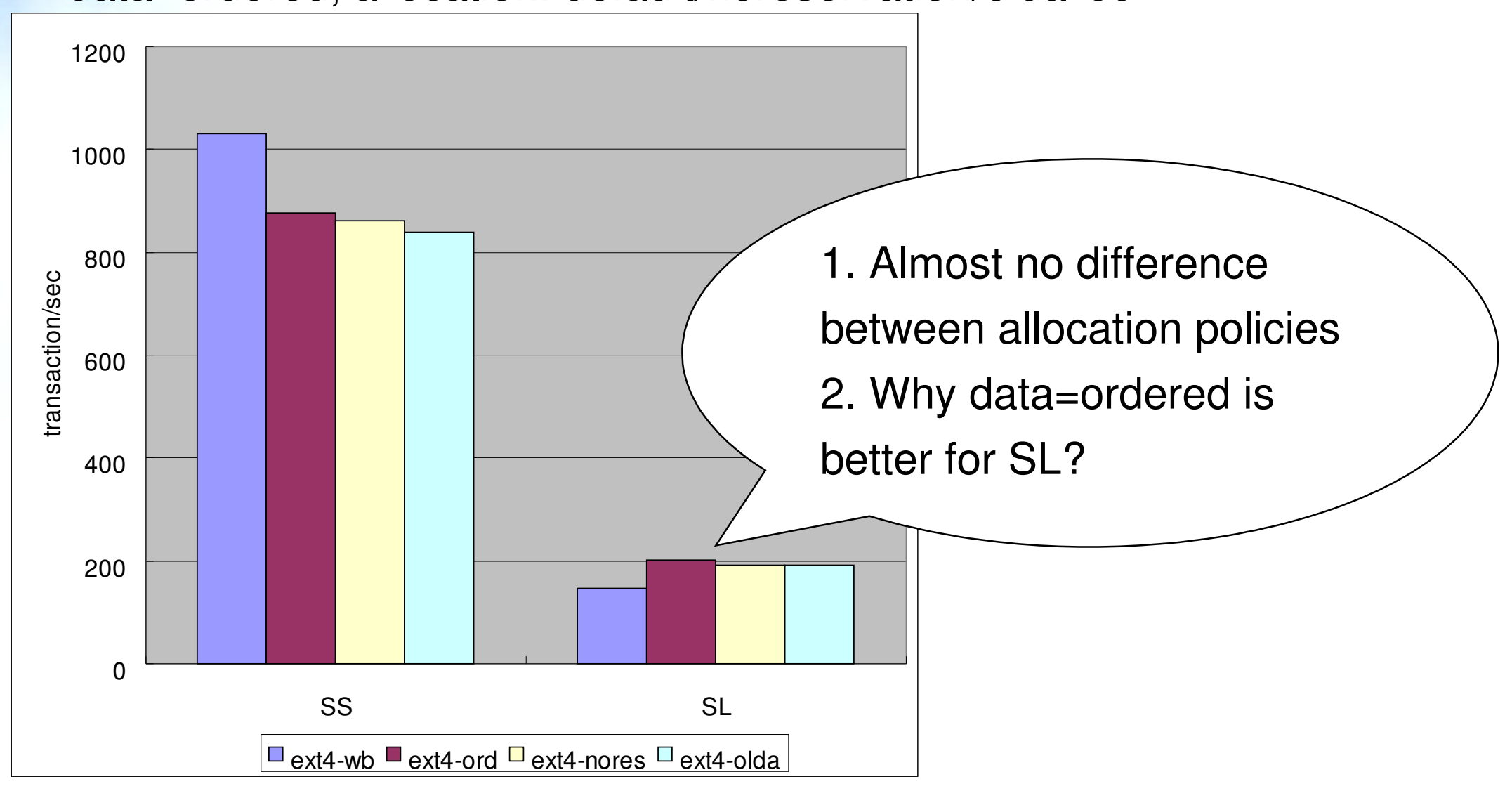




\section{Case study - btrfs}

- Condition

- Block size: 4k/16k, allocation: ssd option on/off

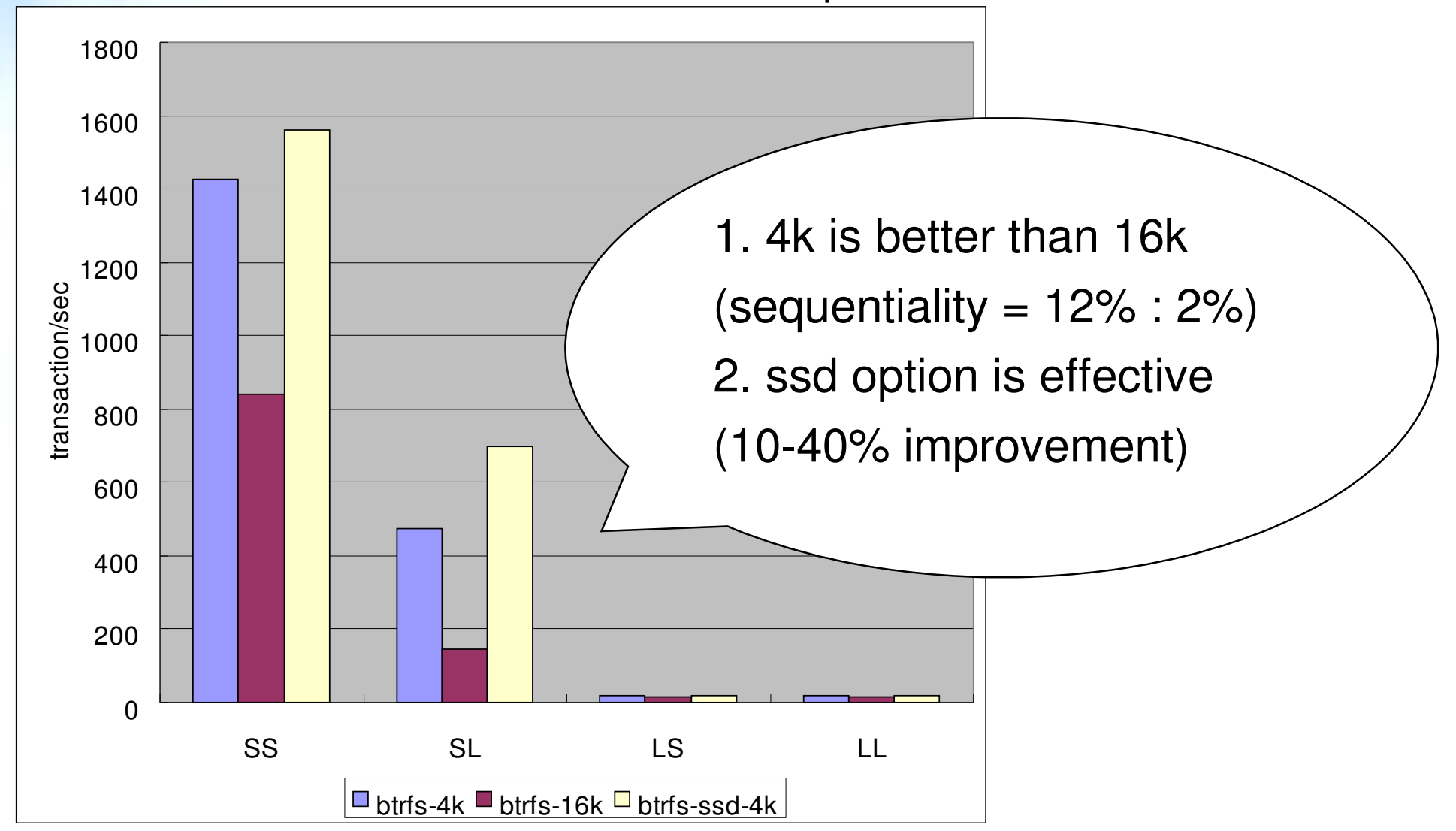




\section{Case study - xfs}

- Condition

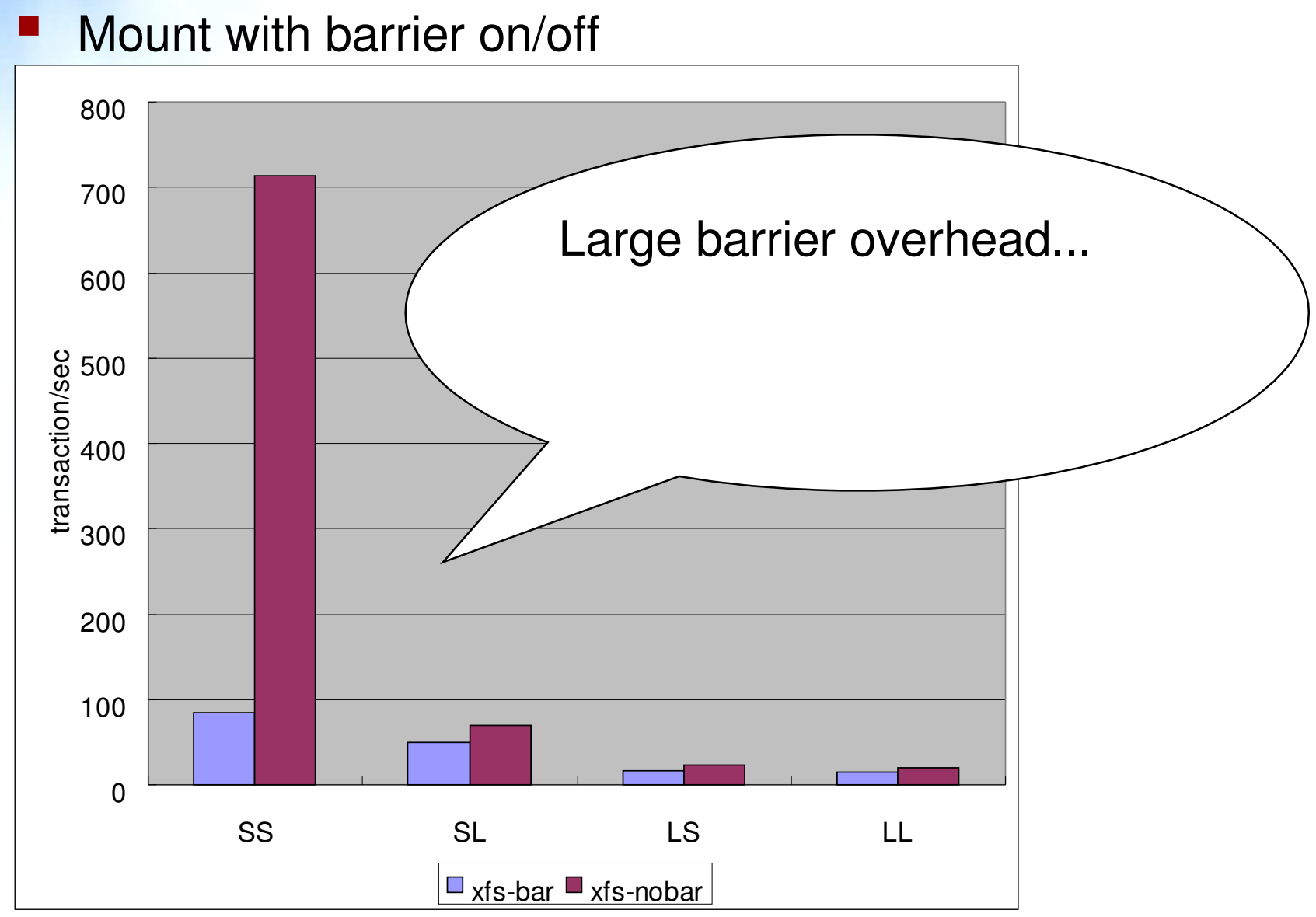




\section{Design consideration for SSD}

- Lessons from flash FS (ex. logfs)

- Sequential writing at multiple logging points

- Wandering tree

- Trace-off between sequentiality vs. amount of write

- Cf. space map (Sun ZFS)

- Need to optimize garbage collection overhead

- Either FS itself or FTL in SSD

- Next topic: End-to-end optimization

- Exchange info with SSD (trim, SSD identification)

- Make best use of parallelism 


\section{New interfaces for SSD (t13.org)}

- Trim command

- Let device know which LBA range is not used

- This will be helpful for optimizing FTL

- Should be passed through: FS $\rightarrow$ bio $\rightarrow$ scsi $\rightarrow$ libata

- Passing bio with no data

- What about $\mathrm{I} / \mathrm{O}$ reordering \& $\mathrm{I} / \mathrm{O}$ queuing?

- SSD identification (added to "ATA identify")

- Report size of page and erasable block

- Physical or effective?

- Useful for FS and volume manager 


\section{Parallel processing of small I/O}

- Make better use of $\mathrm{I} / \mathrm{O}$ queuing (TCQ or NCQ)

- Parallel processing of small $1 / O$

- Desktop environment? Barrier?

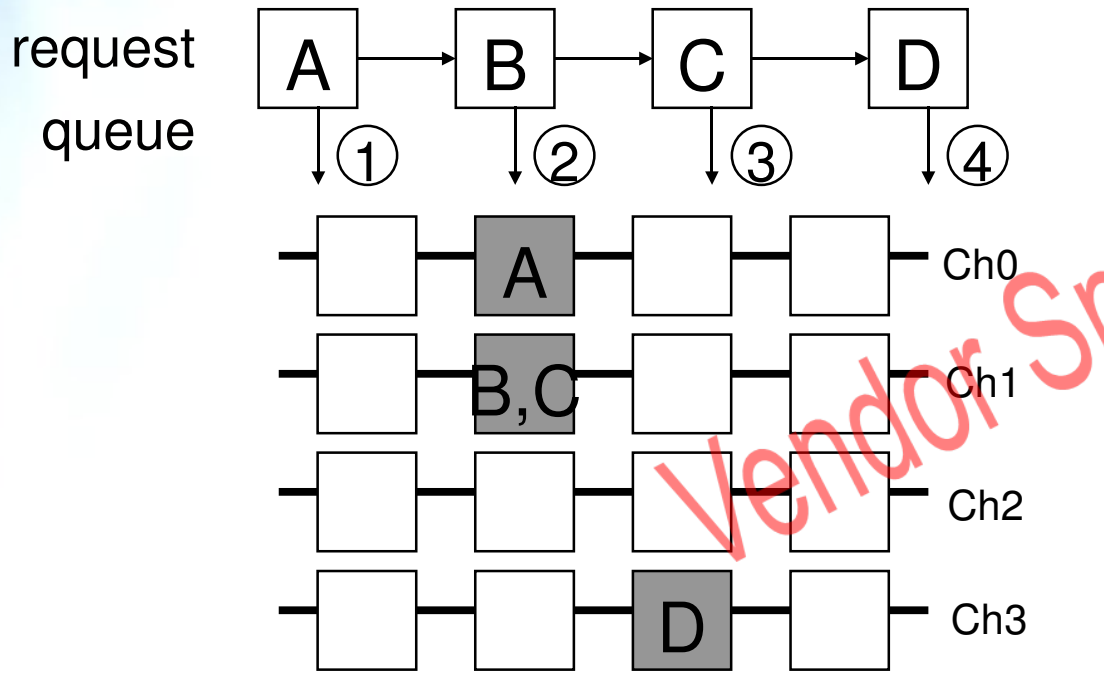

without I/O queuing, 4 steps

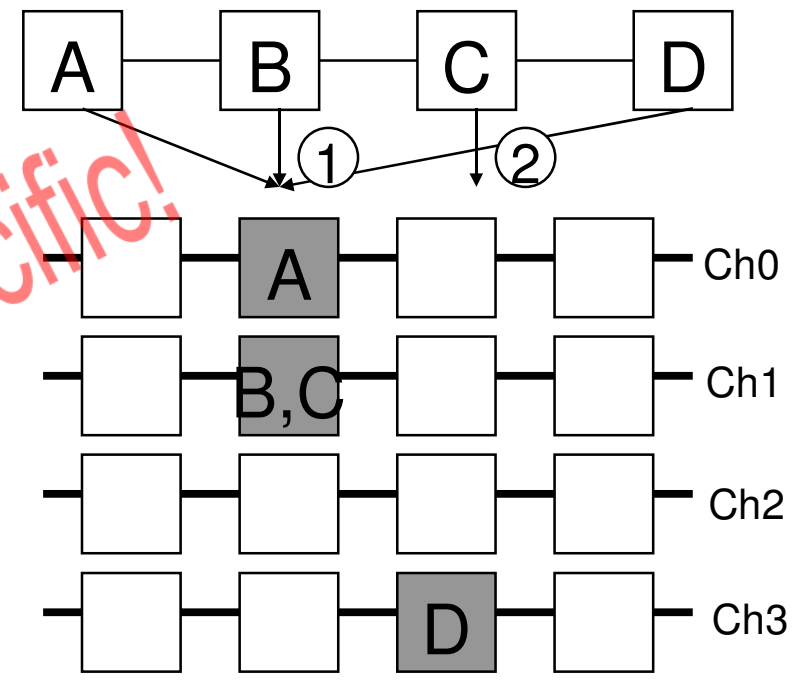

with I/O queuing, 2 steps 


\section{Summary}

- Optimization for SSD

- Alignment is important

- Segmented sequentiality

- Make better use of parallelism (either small or large)

- I/O barrier may stall the pipelined processing

- What can you do?

- File system: alignment, allocation policy, design (ex. COW)

- Block layer: bio w/ hint, barrier, I/O queueing, scheduler(?)

- Volume manager: alignment, allocation

- Virtual memory: read-ahead 


\section{References}

- T13 spec for SSD

- http://www.t13.org/documents/UploadedDocuments/docs2007/e07153rc

- http://www.t13.org/documents/UploadedDocuments/docs2007/e07154rc

- Introduction to SSD and flash memory

- http://download.microsoft.com/download/a/f/d/afdfd50d-6eb9-425e-84e1

- http://download.microsoft.com/download/d/f/6/df6accd5-4bf2-4984-8285

- http://download.microsoft.com/download/a/f/d/afdfd50d-6eb9-425e-84e1

- FTL description \& optimization

- BPLRU: A Buffer Management Scheme for Improving Random Writes in Flash Storage (FAST '08) 


\section{Appendix. I/O Pattern}

- SS workload - ext4, xfs
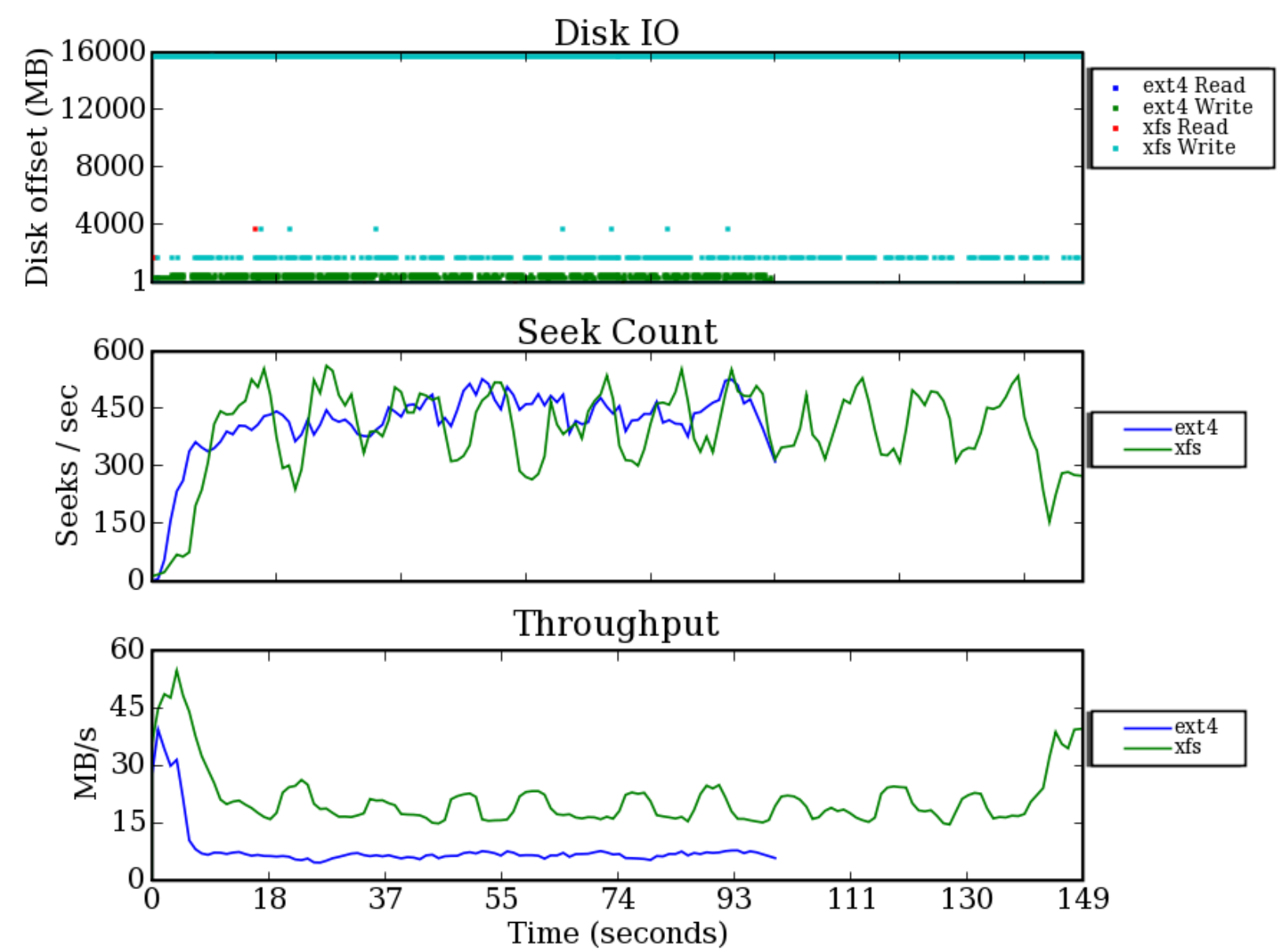


\section{Appendix. I/O Pattern}

- SS workload - btrfs, nilfs
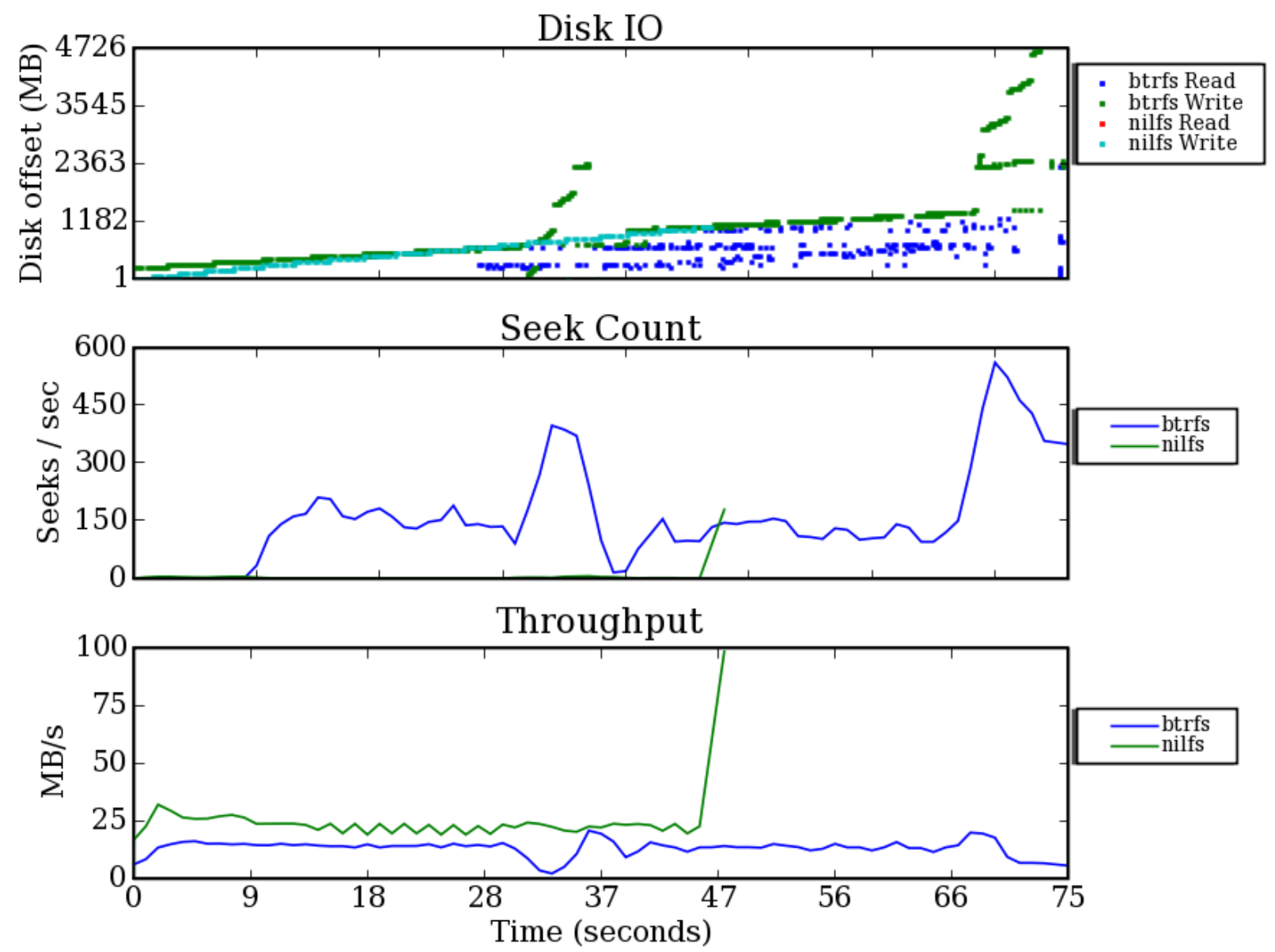


\section{Appendix. I/O Pattern}

- SL workload - ext4, xfs
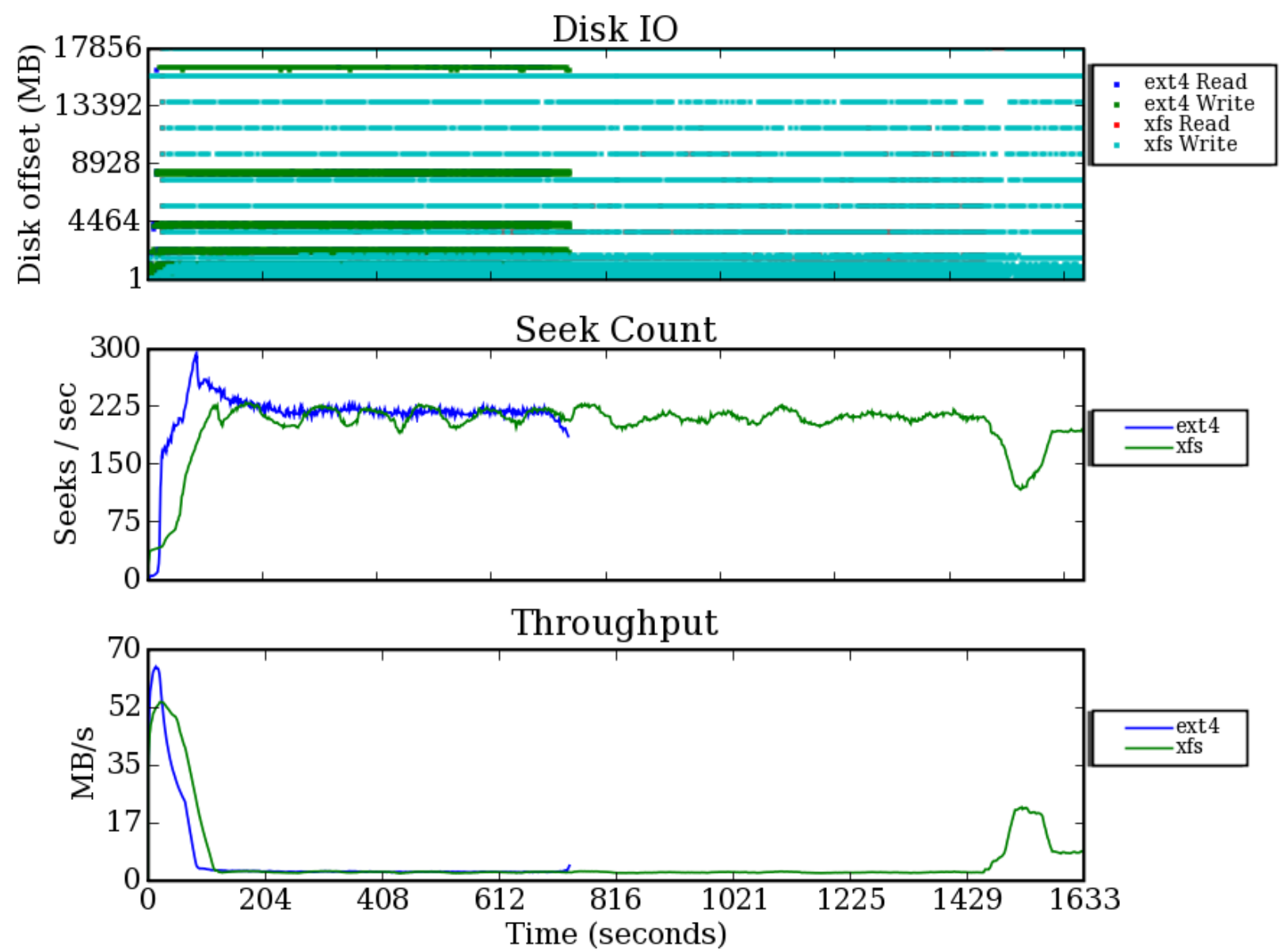


\section{Appendix. I/O Pattern}

- SL workload - btrfs, nilfs
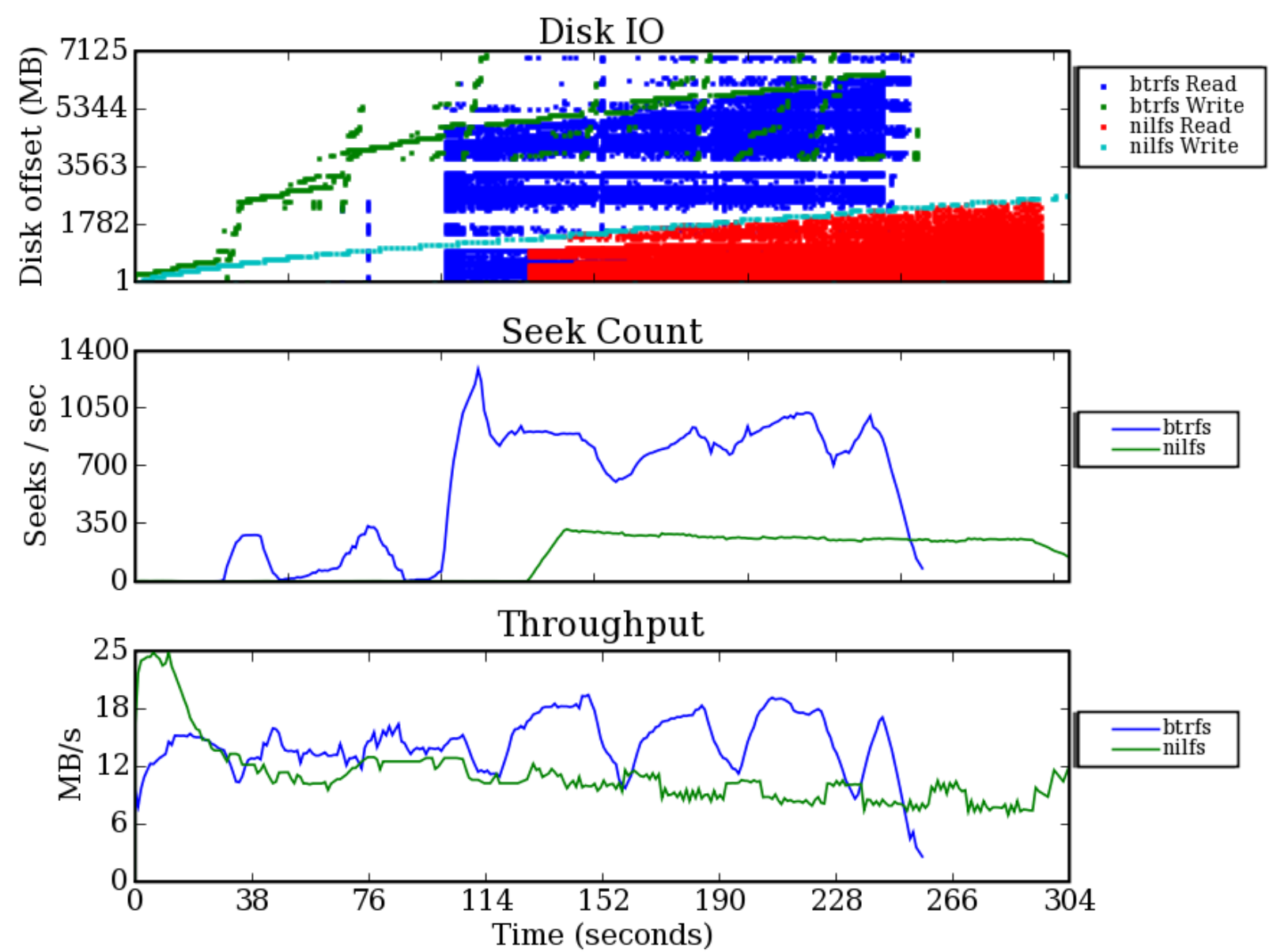


\section{Appendix. I/O Pattern}

- LS workload - ext4, reiserfs, xfs
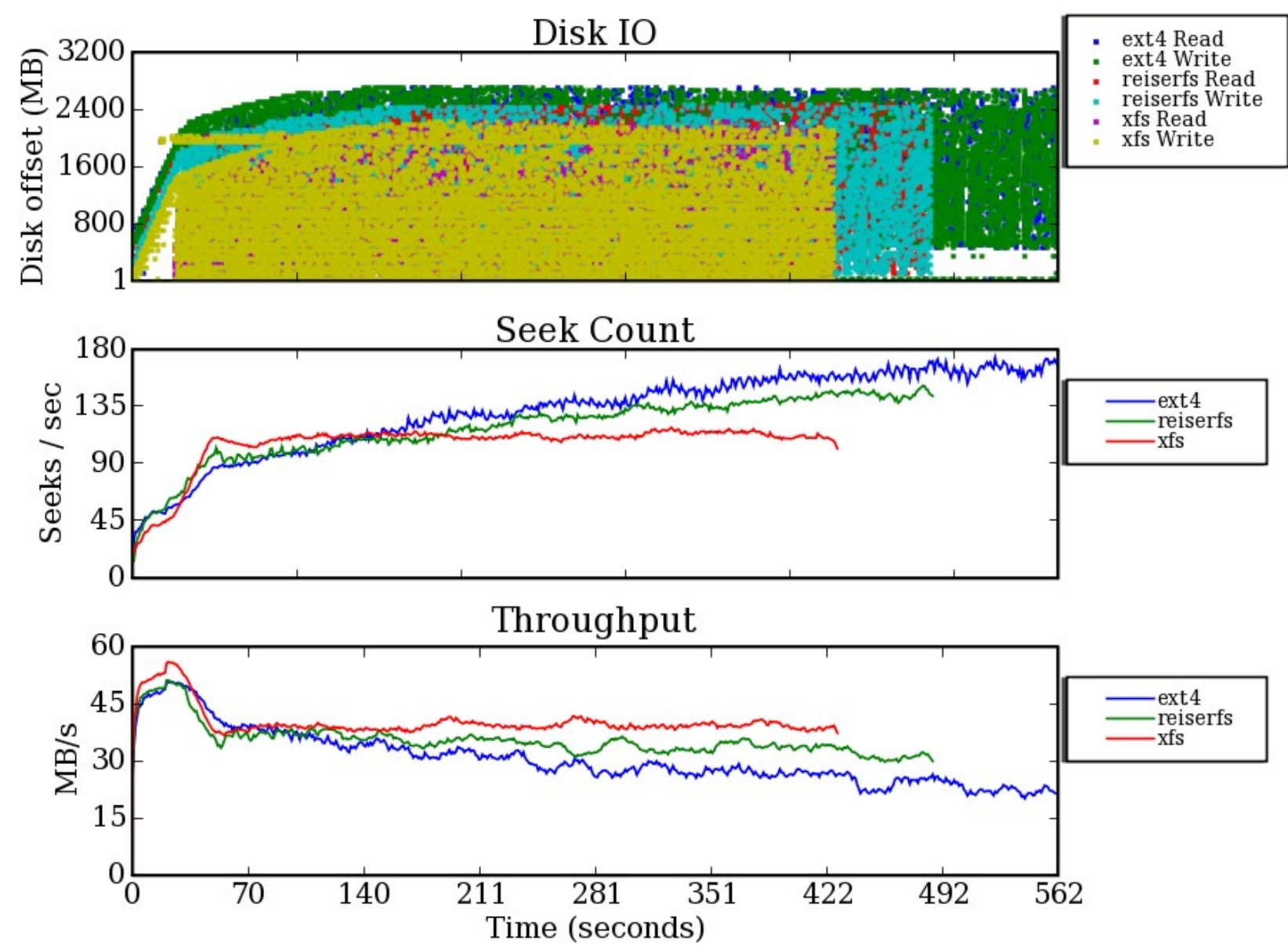


\section{Appendix. I/O Pattern}

- LS workload - btrfs, nilfs
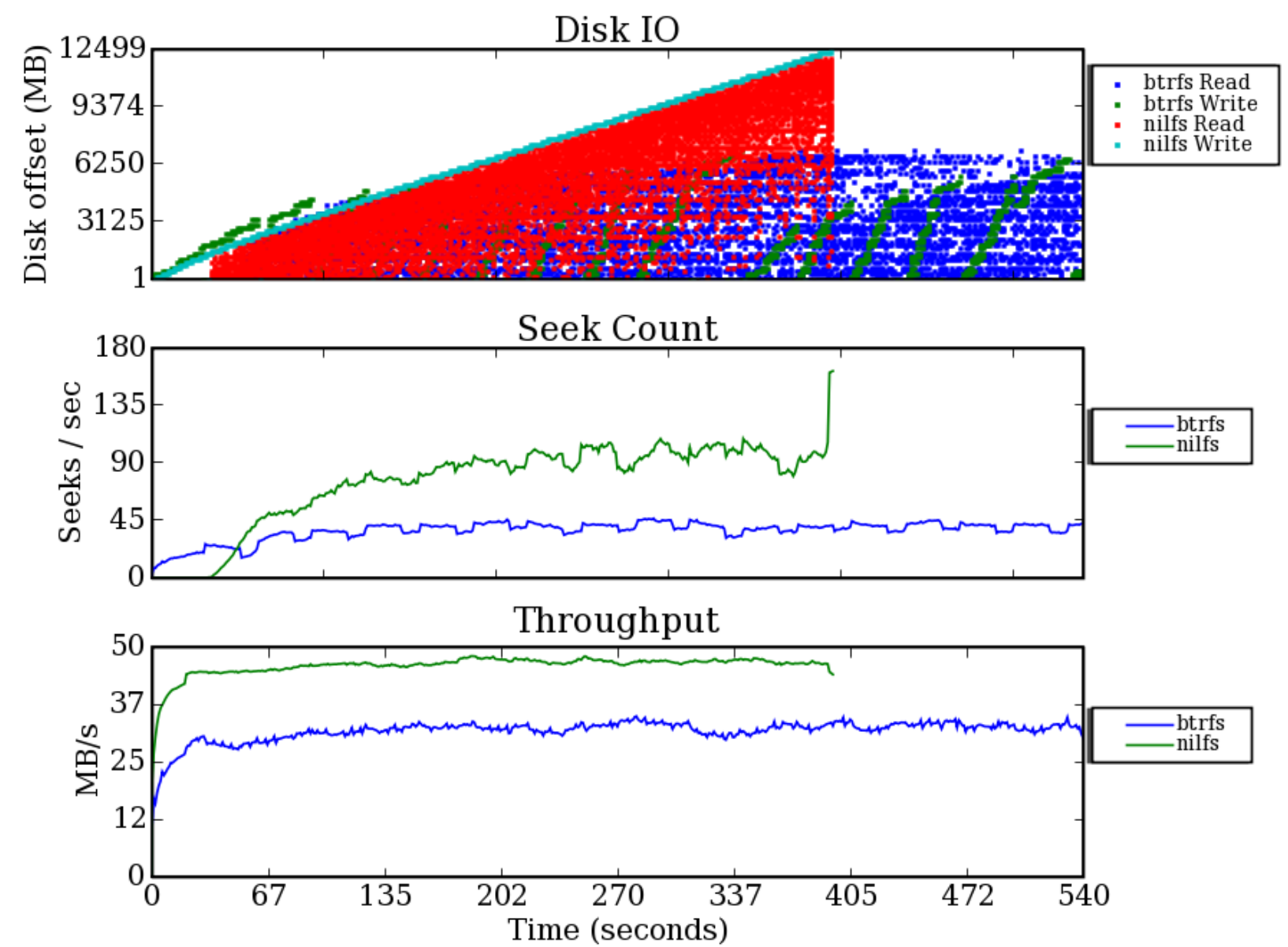


\section{Appendix. I/O Pattern}

- LL workload - ext4, reiserfs, xfs
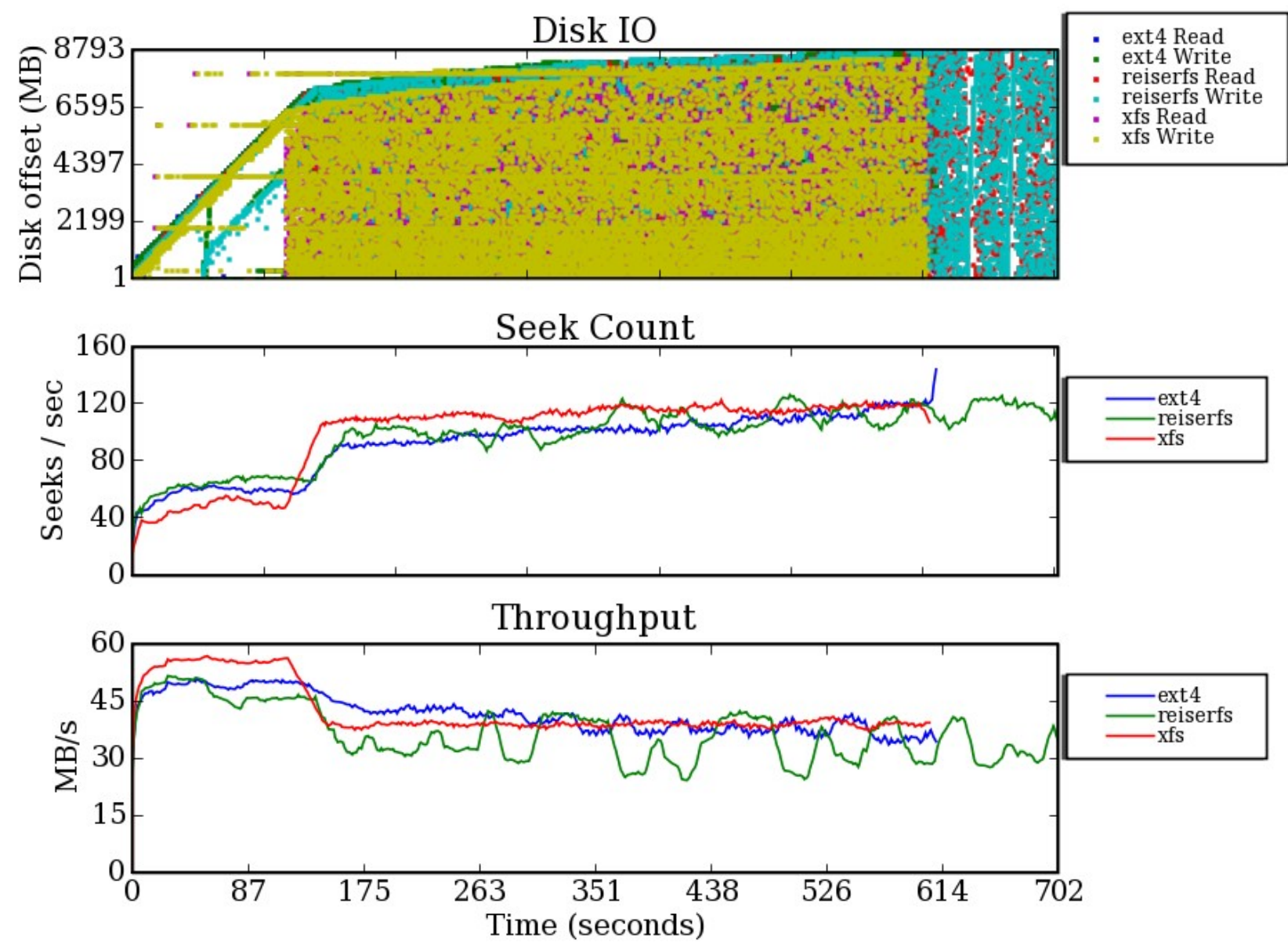


\section{Appendix. I/O Pattern}

- LL workload - btrfs, nilfs
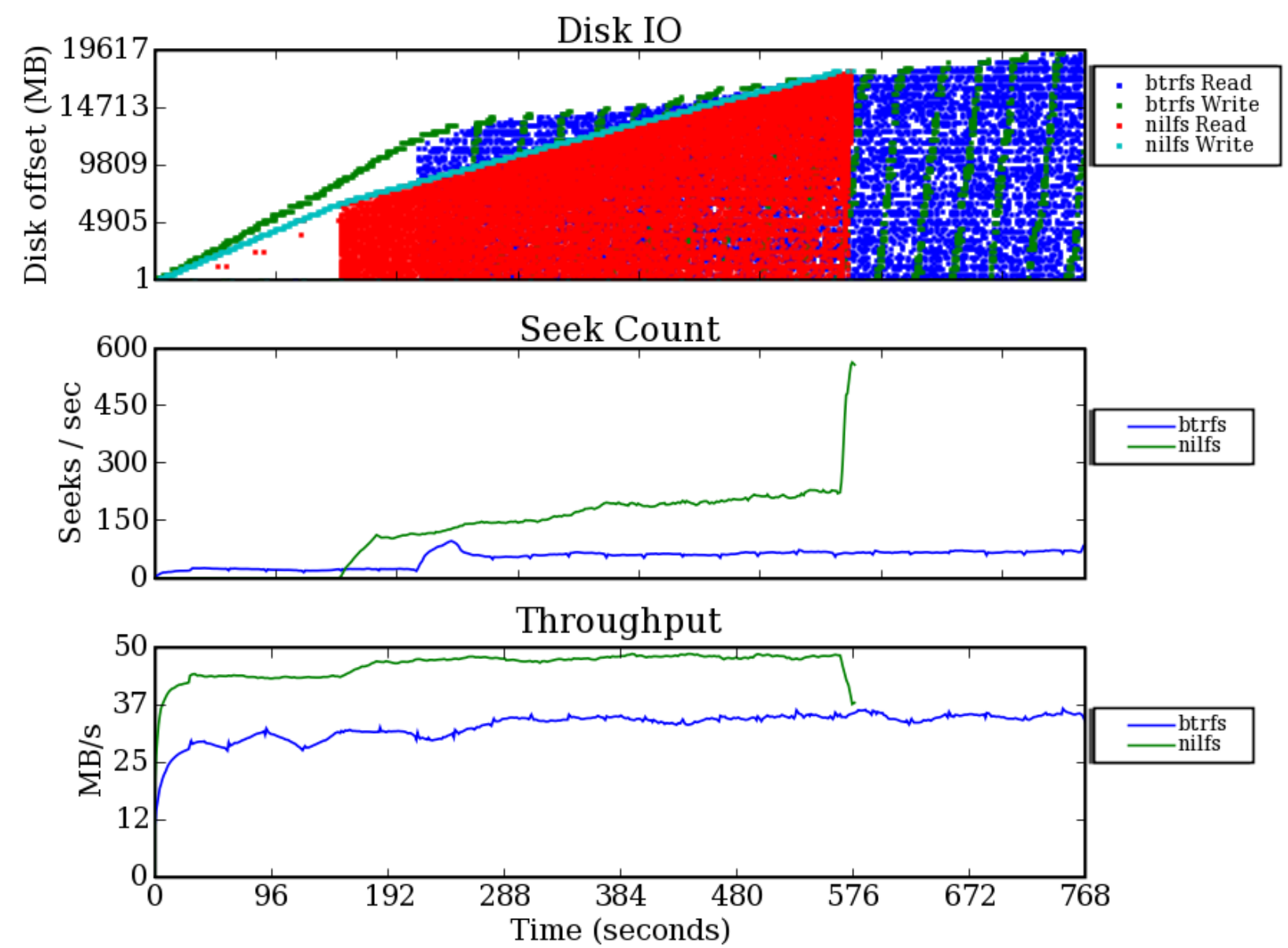